
\title{
25 Research Suare \\ Bioinspired Polypyrrole based Fibrillary Artificial Muscle with Actuation and Intrinsic Sensing Capabilities
}

\section{Mihaela Beregoi}

National Institute of Materials Physics

\section{Samuel Beaumont}

Technical University of Cartagena

Alexandru Evanghelidis

National Institute of Materials Physics

\section{Toribio F. Otero}

Technical University of Cartagena

lonut Enculescu ( $\nabla$ encu@infim.ro)

National Institute of Materials Physics

\section{Research Article}

Keywords: polypyrrole, electrospinning, microribbons, fibrillary networks, mechanical sensor, artificial muscle

Posted Date: November 30th, 2021

DOI: https://doi.org/10.21203/rs.3.rs-1051116/v1

License: (c) (1) This work is licensed under a Creative Commons Attribution 4.0 International License. Read Full License 


\section{Abstract}

Artificial muscles comprise a bunch of materials, composites and devices performing a similar behavior to biological muscles, since a mechanical actuation is produced while consuming a certain amount of energy. However, in order to mimic the multiple simultaneous functionalities of the natural muscles, i.e. the proprioception, new devices should be designed. A non-conventional, bioinspired device based on polypyrrole coated electrospun fibrous microstructures, which works simultaneously as artificial muscle and mechanical sensor is reported. A simple fabrication algorithm based on electrospinning, sputtering deposition and electrochemical polymerization produced electroactive aligned ribbon meshes with analogous characteristics as natural muscle fibers. These can simultaneously produce a movement (by applying an electric current/potential) and sense the effort of holding weights (by measuring the potential/current while holding objects up to $24 \mathrm{mg}$ ). The amplitude of the movement decreases by increasing the load, a behavior similar with natural muscles. Moreover, when different weights were hanged on the device, it senses the load modification, demonstrating a sensitivity of about $6 \mathrm{mV} / \mathrm{mg}$ for oxidation and $3 \mathrm{mV} / \mathrm{mg}$ for reduction. These results are important since simultaneous actuation and sensitivity are essential for complex activity. Such devices with multiple functionalities can open new possibilities of applications as smart prosthesis or lifelike robots.

\section{Introduction}

In the past decade the development of nature inspired soft actuating materials using simple, straightforward techniques has progressively increased. The motivation is the disruptive potential for applications of devices with complex actuation functions similar to those of living organisms. A notable example of this is represented by the field of artificial muscle research where devices fabricated using classical architectures ${ }^{1-3}$ are replaced by faster responding, highly flexible and mechanically stable while simultaneously sensitive materials ${ }^{4,5}$ seek to closely mimic the natural muscles functions. Consequently, various stimuli responsive components were integrated in bioinspired devices such as conducting polymers $^{6,7}$, graphene ${ }^{8}$, carbon nanotubes ${ }^{9}$, dielectric elastomers ${ }^{10}$, etc. and some of them have also sensing abilities. In general, the sensing functions were artificially added to the muscle ${ }^{11-13}$ and therefore few reports about artificial muscles with intrinsic sensing properties ${ }^{4,5,14}$ were presented in scientific literature.

It was demonstrated that artificial muscles with sensing abilities can be fabricated by utilizing conducting polymers, especially polypyrrole (PPy), certain similarities to natural muscles being responsible for this function. A comprehensive comparison between how the natural muscles and those based on PPy behave, can be found in ref. ${ }^{15}$ The electrochemical reactions that generate the movement, more precisely the ionic exchange between the liquid electrolyte and the PPy film emerging from applying a current/potential can be influenced by numerous parameters. It was reported that PPy based bilayer and trilayer actuators can also be used as physical (mechanical ${ }^{16}$ or tactile ${ }^{17,18}$ ) or chemical (current, concentration, temperature, electrolyte type ${ }^{19,20}$ ) sensors. Although some progress in developing 
intrinsically sensing artificial muscles has been made, improvements are still needed for obtaining more reactive, highly sensitive devices suited for applications.

One approach in order to obtain improved performances is to replace the conducting polymer films with fibrous networks for increasing the active surface and flexibility, thus facilitating the ionic exchange and hence generating highly responsive devices. A fabrication way to obtain fibrous materials with some morphological features similar to animal muscles is the electrospinning technique. In a conventional electrospinning setup, a polymer solution is converted into micrometer and submicrometer thin solid fibers by using a high voltage source, a collector, a pumping system, a spinneret and in some cases a controlled environment enclosure. A solution jet is obtained from a droplet by employing an intense electrical field, and while traveling between the needle and the collector the jet solidifies due to solvent evaporation resulting in a fiber ${ }^{21,22}$. This technique allows obtaining random or aligned polymer fiber webs with tailored characteristics which, after functionalization could work as contractile microfibers (similar in diameter with natural myofibrils) and can be packed in parallel bundles (similar with skeletal muscle fibers) ${ }^{23,24}$.

The current work deals with the mechanical sensing properties of a fibrous artificial muscle, which mimics the morphology of natural muscle fibers. The fibrillary structures were obtained through electrospinning and coated with a thin metal layer. Further, the electroactive material (PPy) was deposited onto the metallic layer by electropolymerization in such a way that the PPy covers the microribbons while preserving the morphology and therefore the high active area of the electrospun networks. The actuation and sensing abilities were tested by following the electrochemical response from the artificial muscle in a three-electrode cell while changing several masses hanged to the device. The material shows the potential to update the simple, actuation only, artificial muscles with new fibrous, intrinsic sensitive ones, closer in function to animal muscles.

\section{Results}

\subsection{Artificial muscle actuation behavior}

As described in a previous work ${ }^{25}$, a curling movement is expected during the application of a constant current or potential. On one hand, when the artificial muscle has not any mass attached, an anticlockwise curling movement was observed during the application of a constant anodic potential and a clock-wise curling movement was observed during the application of a constant cathodic potential. The movement was reversible while a square potential signal was applied, showing a more pronounced curling motion at the reduction phase. Figure 1 presents two snapshots from a video recorded while the square potential signal was applied to the material without any mass attached. Figure 1a depicts the end of the movement after reduction, while Figure 1a' shows the end of the movement after oxidation. Video S1 from Supplementary Information shows the full movement during two consecutive periods of the square potential applied. 
When a mass of $6 \mathrm{mg}$ is attached to the micribbon strip, a low amplitude movement is observed, as shown in Video S2. As the attached mass increases the movement completely disappears.

\subsection{Artificial muscle mechanical self-sensing properties}

The intrinsic-sensing capabilities of the artificial muscle were monitored during the actuation using cyclic voltammetry, square waves chronopotentiometry and EIS techniques. Thus, CVs in $0.1 \mathrm{M} \mathrm{LiClO}_{4}$ aqueous solution were recorded while mechanical stress was applied and are presented in Figure $2 \mathrm{a}$. In the CV plots, one pair of anodic/cathodic peaks can be found specific to the oxidation/reduction of PPy film. By hanging different weights on the artificial muscle, the oxidation/reduction potentials shift to more electropositive potentials (a more noticeable process for oxidation). As well, the recorded current peak decreases by increasing the clung mass. A representation of the shifted potentials as a function of the attached mass is plotted in Figure 2(b,c). It should be noted that the results obtained at 24 mg were not take into consideration for estimating the sensor sensitivity because a potential/current saturation appears at this weight.

In Figures 3a and 3c, the anodic and cathodic chronopotentiometric responses while current pulses of \pm 5 $\mathrm{mA}$ for $3 \mathrm{~s}$ are applied are shown for each mass. For comparison purpose, a normalization of the data was done taking as a baseline the potential reached by the material for the first applied cathodic current (Figure 3a) or applied anodic current (Figure 3c).

An increase (shift towards more electropositive values) in potential is observed for both anodic and cathodic responses as the mass rises until a saturation occurs in the case of polymer oxidation. A representation of the potentials recorded versus load during the mechanical stress is presented in Figure $3 \mathrm{~b}$ and $3 \mathrm{~d}$.

The representation of consumed energy while different weights are loaded on the microribbon strip for the oxidation and reduction processes are displayed in Figure $4 \mathrm{a}$ and $4 \mathrm{~b}$. Similar with recorded potential, the energy consumed by the electrochemical reactions increases by rising the load.

In the case of anodic response, no significant potential changes occurs when hanging the 24 mg weight when comparing with $18 \mathrm{mg}$ one, therefore this weight was not taken into consideration for estimating the slopes of potential and consumed energy.

A set of EIS measurements were performed in order to quantify how the load influences a set of specific electrode parameters. It is noticeable that since the so-named PPy film is a bunch of curly polymeric chains, whose actuation is due to the cooperative actuation of every single chain during redox reactions (a deeper explanation is shown in Section 3), there is not a true single double layer because there is not a true single film interface. With this in mind, the recorded EIS experimental data were interpreted by using an approximated electrical equivalent circuit presented in Figure 5c. The circuit consists of series connected elements $\left(R_{s}\right.$ - charge transfer resistance for the electrolyte resistance, $R_{1}$ - charge transfer resistance corresponding to PPy/electrolyte virtual interface, $R_{2}$ - charge transfer resistance of Au/PPy 
virtual interface and $\mathrm{W}_{0}$ - Warburg semi-finite impedance correlated with the diffusional part characteristic to low frequencies) in parallel with two constant phase elements (CPE, appearing as double layer capacitances of the two mentioned interfaces).

In Figure 5a (inset representing a zoom on the semicircles) are presented thee Nyquist plots recorded while hanging different weights (up to $24 \mathrm{mg}$ ) on the artificial muscle. The complex plane plots exhibit one low frequency region assigned to the ions migration described by the Warburg diffusion and one high frequency region related to charge transfer mechanism. By increasing the load, the semicircle radius increases. In Figure $5 b$ the diffusion coefficients computed by using the time constants emerging from fitting the experimental data for each mass are presented.

A large difference between the diffusion coefficient for unloaded artificial muscle and loaded one can be observed. Further a slight change of the diffusion coefficient values appears when increasing the load, while at $24 \mathrm{mg}$ a saturation occurs which is in a good agreement with CV and chonopotentiometry measurements.

\section{Discussion}

When the PPy coated microribbon strips are immersed into an electrolyte solution and a square current or potential signal is applied, $\mathrm{p}$-doping and p-dedoping processes take place i.e. reversible oxidation and reduction of polymeric chains ${ }^{26,27}$. The electrochemical reactions of the polymer induce the conformational movement of the chains, the microscopic cooperative actuation leading to the macroscopic swelling and shrinking of the polymer structure during oxidation and reduction by the subsequent lodging and expelling of electrolyte molecules, respectively ${ }^{28,29}$. In this particular case the fibrous structure of the material i.e. a high aspect ratio architecture leads to a macroscopic curling movement, being anti-clockwise during oxidation and clockwise during reduction (Figure 1a and 1a'). The high speed and large amplitude of these processes are favored by the high exchange active surface of the fibrillary artificial muscle.

The mass load sensitivity of the artificial muscle during actuation was tested. By recording CVs with different masses as a load, it was found that for both anodic and cathodic current peaks' intensities decrease while the corresponding potential becomes more electropositive as the load is increased (Figure 2a). The movement's amplitude gets less visible as more load is added to the structure (Video S2). These changes in specific electrochemical parameters can be attributed to a decrease of the ionic migration. The actuation process is shown as a consequence of energy minimization taking place and therefore by adding a load this process is somehow disturbed, the ionic exchange between the conducting polymer and electrolyte being modified. Simultaneously, a limitation of the ability to actuate of the artificial muscle occurs as the load increases. As is the case for natural muscles, the device can undergo fatigue processes and after a certain weight value is reached it cannot make the difference between the loads. The sensitivity was $6.1 \pm 0.49 \mathrm{mV} / \mathrm{mg}$ (for oxidation, Figure $2 \mathrm{~b}$ ) and $2.7 \pm 0.24 \mathrm{mV} / \mathrm{mg}$ (for reduction, Figure 2c). These results are in a good agreement with the theoretical model developed by Otero's 
research team established for a material constituted by electrochemical reaction-driven molecular machines under galvanostatic conditions. The evolution of the consumed energy by the reaction, and the potential reached (as one of the components of the consumed energy) follow a linear dependence with the load mass, respecting the Equations (1) and (2) 30,31 :

$$
\begin{gathered}
E=C_{1}+C_{2} m \\
U(t)=C_{3}(t)+C_{4}(t) m
\end{gathered}
$$

The experimental potential reached by the artificial muscle at any reaction time can be obtained from the chronopotentiograms previously shown (Figure $\mathbf{3 a}$ and $\mathbf{3 c}$ ) while the experimental energies consumed by the system during the electrochemical reactions can be calculated from the product of the potential reached and the charge consumed at any reaction time ${ }^{32}$. Figures $3 a$ and $3 c$ show, respectively, the potential reached by the artificial muscle after application of a square current wave of $\pm 0.5 \mathrm{~mA}$ for $3 \mathrm{~s}$ correlated with the consumed energy during the electrochemical reactions for each load. Both sensing values fit the theoretical model, obtaining the mechanical sensor calibration lines. The slopes (6.7 \pm 0.48 and $-3.9 \pm 0.38 \mathrm{mV} / \mathrm{mg}$ from Figure $3 \mathrm{~b}$ and $3 \mathrm{~d} ; 3.4 \pm 0.24$ and $1.97 \pm 0.18 \mu \mathrm{J} / \mathrm{mg}$ from Figure $4 \mathrm{a}$ and $4 \mathrm{~b}$ ) represent the sensitivity of the sensor. The calibration lines are similar with those obtained analyzing the peak potential from CVs. From these lines, the weight supported by the fibrillary artificial muscle can be measured. In both cases, a difference in the oxidation and reduction slopes is founded, leading to an energetic asymmetry. This effect has been justified considering the different types of macroscopic volume changes of the polymer (swelling-relaxation consumes a different amount of energy than shrinking-compaction) and was expected from the actuation results by observing the extension of the curling movement. When there are no attached loads, the oxidation process tends to be more energetically favored (Figure 3c), leading to deep curling movements (Figure 1a). The asymmetry is also due to the exchange of solvent molecules to balance the osmotic pressure ${ }^{33}$.

The effect of the load modification on the ionic migration processes was followed by EIS. The weak migration of the electrolyte molecules (associated with minimization of diffusive processes and charge transfer resistance increase) at high load was demonstrated by estimating the electrode parameters for EIS measurements (displayed in Table 1). Thus, it was found that the electrolyte resistance (Rs) slowly increases by adding masses. As well, no significant changes in the charge transfer resistance and double layer capacitance of PPy/electrode interface $\left(\mathrm{R}_{2}\right.$ and $\left.\mathrm{CPE}_{2}\right)$ under mechanical stress appear. In contrast, an important difference between the charge transfer resistance and double layer capacitance of PPy/electrolyte interface $\left(R_{1}\right.$ and $\left.C P E_{1}\right)$ obtained with or without added mass can be identified (for $R_{1}$ being almost 3 times higher for maximum load than unloaded muscle and almost 7 times higher for $\mathrm{CPE}_{1}$ ). Likewise, analyzing the $\mathrm{n}$ factor, it seems that the electrode crosses from a Warburg to a capacitor behavior by rising the load. These mean that the incorporation of electrolyte ions into PPy film decreases 
by increasing the load. Moreover, a diffusion coefficient (D) for each weight was estimated considering a geometrical model (Equation 3) suitable for a conducting polymer film with a finite thickness $(L)$ and an ionic diffusion time constant $(\tau)^{34}$.

$$
\tau=\frac{L^{2}}{\mathrm{D}}
$$

\section{Table 1}

Electrode parameters for each weight clung on the artificial muscle.

\begin{tabular}{|llllll|}
\hline Element & $\mathbf{0 ~} \mathbf{~ g}$ & $\mathbf{6} \mathbf{~ m g}$ & $\mathbf{1 2} \mathbf{~ m g}$ & $\mathbf{1 8} \mathbf{~ m g}$ & $\mathbf{2 4} \mathbf{~ m g}$ \\
\hline $\mathrm{R}_{1}, \Omega$ & 60 & 67 & 101 & 133 & 164 \\
\hline $\mathrm{CPE}_{1}, \mathrm{~S}$ & 0.00081 & 0.00021 & 0.00015 & 0.00013 & 0.00012 \\
$\mathrm{n}$ & 0.57 & 0.74 & 0.77 & 0.77 & 0.77 \\
\hline $\mathrm{\tau}, \mathrm{s}$ & 0.002 & 0.18 & 0.22 & 0.26 & 0.29 \\
$\mathrm{D}\left(\mathrm{cm}^{2} / \mathrm{s}, 10^{-10}\right)$ & 845 & 9.4 & 7.7 & 6.5 & 5.8 \\
\hline
\end{tabular}

The large difference between the estimated diffusion coefficients of unloaded and loaded artificial muscle lead to the idea that an important part of the muscle mechanical sensitivity can be attributed to electrolyte ion migration, the process being reduced by increasing the load. As expected, this ionic inhibition causes also a decrease of movement amplitude, until a saturation occurs when no motion is observed.

\section{Conclusions}

A fiber-based architecture of artificial muscles consisting of electrospun microribbons covered with polypyrrole was found to have intrinsic mechanical sensing capabilities, thus being close to both the morphology and functionality of natural animal muscles. The reversible electrochemical reactions of polypyrrole while immersed in a $\mathrm{LiClO}_{4}$ electrolyte under galvanostatic control lead to both actuation and sensing functionality. A reversible anti-clockwise/clockwise curling movement is observed for this particular structure - the actuation function. When different masses were employed as loads for the artificial muscle, mechanical sensing properties were found - the sensing function. Thus, specific electrochemical parameters were depending on the load until a saturation occurs (in this particular case a mass of $24 \mathrm{mg}$ ), fitting the theoretical model previously developed. The mechanical sensitivity of the artificial muscles was found to be approximately $6 \mathrm{mV} / \mathrm{mg}$ for oxidation and $3 \mathrm{mV} / \mathrm{mg}$ for reduction in 0 $18 \mathrm{mg}$ weight range. These artificial muscle structures denote their potential as constitutive materials for a new generation of dual actuator-sensor soft electrochemical devices. This added function will allow complex machines to execute finely tuned tasks which need appropriate feedback from the sensorial 
system without the need of an external sensor monitor. This would update the former actuation-only artificial muscles towards new fibrillary self-sensing ones, coming closer to animal muscles.

\section{Methods}

\section{Materials and equipments.}

For preparing the electrospun microribbon nets, nylon 6/6 (pellets, Sigma Aldrich, St. Louis, MO, USA; CAS number 32131-17-2) and formic acid (ACS reagent, $\geq 88.0 \%$, Sigma Aldrich, St. Louis, MO, USA; CAS number 64-18-6) were used. Further, the conducting polymer was synthesized using lithium perchlorate ( $\mathrm{LiClO}_{4}$, battery grade, dry, 99.99\% metals basis, Aldrich, St. Louis, MO, USA; CAS number 7791-03-9), pyrrole (for synthesis, Sigma-Aldrich, St. Louis, MO, USA; CAS number 109-97-7), and acetonitrile (for HPLC, gradient grade, $\geq 99.9 \%$, Honeywell, Charlotte, NC, USA; CAS number 75-05-8) as solvent. A gold target (99.99\% purity, Kurt J. Lesker) and $\operatorname{Ar}(99.9999 \%$ purity, Linde) were employed for the metallization process. All solutions were prepared by using ultrapure water generated in a Milli-Q equipment (18.2 M $\Omega$ $\mathrm{cm})$.

The procedure to cover the metalized microribbons with PPy, involved the use a Parstat 2273 Princeton Applied Research for electropolymerizing the monomer. The metalized microribbons attached on stainless steel frames were used as working electrode (WE), a platinum mesh as counter electrode (CE) and a commercial saturated calomel electrode as reference electrode (RE). For the electrochemical characterization, a MultiAutolab M101 potentiostat/galvanostat equipped with NOVA 2.1.4 software was used. The electrochemical cell was based on strips taken from the PPy coated microribbon net and used as $\mathrm{WE}$, a platimum plate used as $\mathrm{CE}$ and a commercial $\mathrm{Ag} / \mathrm{AgCl}$ electrode as $\mathrm{RE}$. For the metallization process, a Torr DC sputtering equipment was employed.

\section{Preparation of PPy based fibrillary artificial muscle.}

The PPy based fibrillary artificial muscles were prepared according to a procedure described in a previous work $^{25}$. At first, nylon 6/6 microribbons were prepared from a precursor solution by electrospinning in controlled environmental conditions and collecting on copper frames. In a second step, the electrospun ribbons were covered with a thin layer of gold by sputtering and later submerged into a monomeric solution, consisting in $0.2 \mathrm{M}$ pyrrole and $0.1 \mathrm{M} \mathrm{LiClO}_{4}$ in acetonitrile and $2 \%$ water. Then, the metalized nets were used as working electrodes in the setup for potentiostatic electropolymerization of pyrrole, by applying a constant potential of $0.872 \mathrm{~V}$ for $120 \mathrm{~s}$.

\section{Characterization.}


After preparation, the PPy coated microribbon webs were cut in rectangular strips of $1 \times 2 \mathrm{~cm}$ width $\mathrm{x}$ length and submerged in a $0.1 \mathrm{M} \mathrm{LiClO}_{4}$ aqueous solution. Then, using the microribbon strips as electrodes cyclic voltammetry (CV) experiments were performed using -0.60 and $+0.60 \mathrm{~V}$ as potential limits and a scan rate of $100 \mathrm{mVs}^{-1}$. Several cycles were performed allowing the system to remove a possible previous material memory and promoting the exchange of chloride from the electrolyte ${ }^{35-37}$. When a stationary response is obtained, the material's electroactivity data is stored as a reference before the experimental series. The actuation performances of the artificial muscle were tested through cyclic voltammetry experiments considering the same parameters as those mentioned above. In a further step the sensing properties of the artificial muscle were investigated by recording consecutive CVs while sweeping the potential between -0.60 and $+0.60 \mathrm{~V}$ at a scan rate of $100 \mathrm{mVs}^{-1}$, while various weights were clung to the fibrillary strip (as can be seen in Figure 6). Likewise, an anodic and a cathodic chronoamperometric response were obtained when the artificial muscle was subjected to successive square current waves by applying a constant current of $\pm 0.5 \mathrm{~mA}$ for $3 \mathrm{~s}$ each. The square current pulses were repeatedly applied while attaching the masses to the microribbon strip. Corresponding results were acquired by recording the electrochemical impedance spectroscopy (EIS) plots, EIS being a suitable technique for describing the ionic diffusion processes, when different loads were hung on the artificial muscle. The ionic diffusion behavior was investigated by applying a polarization potential of $-0.20 \mathrm{~V}$, in the $0.1-100000 \mathrm{~Hz}$ frequency range, at 10 points per decade and an AC voltage amplitude of $10 \mathrm{mV}$. The experimental data were interpreted by using a ZView 2 (Scribner Assoc.) fitting program, considering an electrical equivalent circuit (Figure 5c) which was also employed to estimate the electrode parameters. The weights used during the experiment were prepared by cutting a copper wire and bending it in a Sshape in order to be attached to the artificial muscle. Since every cut wire had a weight of $6 \mathrm{mg}$, up to four masses were consecutively hanged leading to masses of $6,12,18$ and $24 \mathrm{mg}$ respectively. The first mass was attached to the microribbons by using a piece of adhesive tape, Figure 6 showing the experimental design used during experiments.

\section{Declarations}

\section{Acknowledgements}

This work was supported by the Core Program PN19-03 (contract no. 21 N/08.02.2019) and PN-III-P1-1.1PD-2019-1066. Also, this work was supported by Seneca Foundation (19253/PI/14).

\section{Data availability statement}

The datasets generated and analyzed during the current study are available from the corresponding author on reasonable request.

\section{Author contributions statement}


M.B. covered the microribbon meshes with gold and polypyrrole and along with S.B. performed the electrochemical characterization and data analysis. As well, M.B. and S.B. wrote the initial version of the manuscript. I.E. along with T.F.O. supervised the experiments, interpreted the experimental data and elaborated the final version of the manuscript. A.E. prepared the electrospun microribbons. All authors reviewed the manuscript.

\section{References}

1. Christophersen, M., Shapiro, B. \& Smela, E. Characterization and modeling of PPy bilayer microactuators - Part 1. Curvature. Sens. Actuator B-Chemical, 115, 596-609 (2006).

2. Otero, T. \& Sansinena, J. Bilayer dimensions and movement in artificial muscles. Bioelectrochem. Bioenerg, 42, 117-122 (1997).

3. Hagler, J. R., Peterson, B. N., Murphy, A. R. \& Leger, J. M. Performance of silk-polypyrrole bilayer actuators under biologically relevant conditions. J. Appl. Polym. Sci, 136, 46922 (2019).

4. Lo, C. Y. et al. Highly stretchable self-sensing actuator based on conductive photothermallyresponsive hydrogel.Mater. Today(2021); 10.1016/j.mattod.2021.05.008.

5. Harjo, M. Polypyrrole-coated fiber-scaffolds: Concurrent linear actuation and sensing. J. Appl. Polym. Sci, 137, 48533 (2020).

6. Harjo, M., Jarvekulg, M., Tamm, T., Otero, T. F. \& Kiefer, R. Concept of an artificial muscle design on polypyrrole nanofiber scaffolds., 15, 1-16 (2020).

7. Wang, F., Li, Q., Park, J. O., Zheng, S. \& Choi, E. Ultralow voltage high-performance bioartificial muscles based on ionically crosslinked polypyrrole-coated functional carboxylated bBacterial cellulose for soft robots. Adv. Funct. Mater, 31, 2007749 (2021).

8. Chen, L. Graphene-based actuator with integrated-sensing function. Adv. Funct. Mater, 29, 1806057 (2019).

9. Zheng, W. Artificial muscles based on polypyrrole/carbon nanotube laminates. Adv. Mater, 23, 29662970 (2011).

10. Bruschi, A., Donati, D. M., Choong, P., Lucarelli, E. \& Wallace, G. Dielectric elastomer actuators, neuromuscular interfaces, and foreign body response in artificial neuromuscular prostheses: A review of the literature for an in vivo application. Adv. Healthc. Mater, 10, 2100041 (2021).

11. Kanno, R., Watanabe, S., Shimizu, K. \& Shintake, J. Self-sensing McKibben artificial muscles embedded with dielectric elastomer sensor. IEEE Robot. Autom. Lett, 6, 6274-6280 (2021).

12. Kwak, B. \& Bae, J. Integrated design and fabrication of a conductive PDMS sensor and polypyrrole actuator composite. IEEE Robot. Autom. Lett, 5, 3758-3765 (2020).

13. Yun, S., Jeong, J., Mun, S. \& Kyung, K. U. A highly stretchable optical strain sensor monitoring dynamically large strain for deformation-controllable soft actuator. Smart Mater. Struct, 30, 105020 (2021). 
14. Martinez, J. G. \& Otero, T. F. Three electrochemical tools (motor-sensor-battery) with energy recovery work simultaneously in a trilayer artificial muscle. Electrochim. Acta, 294, 126-133 (2019).

15. Otero, T. F. Towards artificial proprioception from artificial muscles constituted by self-sensing multistep electrochemical macromolecular motors. Electrochim. Acta, 366, 137576 (2021).

16. Shoa, T. Electromechanical coupling in polypyrrole sensors and actuators. Sens. Actuator A-Phys, 161, 127-133 (2010).

17. Otero, T. \& Cortes, M. A sensing muscle. Sens. Actuator B-Chemical, 96, 152-156 (2003).

18. Otero, T. \& Cortes, M. Artificial muscles with tactile sensitivity. Adv. Mater, 15, 279-282 (2003).

19. Otero, T. F. Electroactive macromolecular motors as model materials of ectotherm muscles. RSC Adv, 11, 21489-21506 (2021).

20. Khuyen, N. Q. et al. Multifunctionality of polypyrrole polyethyleneoxide composites: Concurrent sensing, actuation and energy storage. Polymers 12, 2060 (2020); 10.3390/polym12092060

21. Agarwal, S., Greiner, A. \& Wendorff, J. H. Functional materials by electrospinning of polymers. Prog. Polym. Sci, 38, 963-991 (2013).

22. Bhardwaj, N., Kundu, S. C. \& Electrospinning A fascinating fiber fabrication technique. Biotechnol. $A d v, 28,325-347$ (2010).

23. Feher, J. Quantitative human physiology, book subchapter Contractile mechanisms in skeletal muscle (ed. Feher, J.) 249-258 (Academic Press, 2012); 10.1016/C2009-0-64018-6

24. Gu, B. K. A Linear Actuation of Polymeric Nanofibrous Bundle for Artificial Muscles. Chem. Mater, 21, 511-515 (2009).

25. Beregoi, M., Evanghelidis, A., Diculescu, V. C., lovu, H. \& Enculescu, I. Polypyrrole actuator based on electrospun microribbons. ACS Appl. Mater. Interfaces, 9, 38068-380759 (2017).

26. Tobergte, D. R. \& Curtis, S. Handbook of conducting polymers; $10.1017 /$ CBO9781107415324.004 (2013)

27. MacDiarmid, A. G., Mammone, R. J., Kaner, R. B. \& Porter, S. J. The concept of 'doping' of conducting polymers: The role of reduction potentials. Phil. Trans. R. Soc. Lond. A, 314, 3-15 (1985).

28. Beaumont, S. \& Otero, T. F. Chemical sensors from the cooperative actuation of multistep electrochemical molecular machines of polypyrrole: potentiostatic study. Trying to replicate muscle's fatigue signals. Smart Mater. Struct, 27, 074001 (2018).

29. Beaumont, S. \& Otero, T. F. A potentiostatic/galvanostatic study and theoretical description of polypyrrole film electrodes: A model of the intracellular matrix of ectothermic muscle cells. ChemElectroChem, 4, 3091-3099 (2017).

30. Otero, T. F. \& Martinez, J. G. Physical and chemical awareness from sensing polymeric artificial muscles. Experiments and modeling. Prog. Polym. Sci, 44, 62-78 (2015).

31. Otero, T. F., Sanchez, J. J. \& Martinez, J. G. Biomimetic dual sensing-actuators based on conducting polymers. Galvanostatic theoretical model for actuators sensing temperature. J. Phys. Chem. B, 116, 5279-5290 (2012). 
32. Otero, T. F. \& Beaumont, S. The cooperative actuation of multistep electrochemical molecular machines senses the working temperature: voltammetric study. Electrochim. Acta, 257, 403-411 (2017).

33. Otero, T. F. \& Beaumont, S. The energy consumed by electrochemical molecular machines as selfsensor of the reaction conditions: Origin of sensing nervous pulses and asymmetry in biological functions. ChemElectroChem, 5, 3335-3347 (2018).

34. Beregoi, M., Evanghelidis, A., Matei, E. \& Enculescu, I. Polyaniline based microtubes as buildingblocks for artificial muscle applications. Sens. Actuator B-Chemical, 253, 576-583 (2017).

35. Otero, T. F., Martinez, J. G., Fuchiwaki, M. \& Valero, L. Structural electrochemistry from freestanding polypyrrole films: Full hydrogen inhibition from aqueous solutions. Adv. Funct. Mater, 24, 1265-1274 (2014).

36. Otero, T. F. \& Beaumont, S. Chemical sensors from the cooperative actuation of multistep electrochemical molecular machines of polypyrrole: Voltammetric study. Sens. Actuator B-Chemical, 253, 958-966 (2017).

37. Otero, T. F., Alfaro, M., Martinez, V., Perez, M. A. \& Martinez, J. G. Biomimetic structural electrochemistry from conducting polymers: Processes, charges, and energies. Coulovoltammetric results from films on metals revisited. Adv. Funct. Mater, 23, 3929-3940 (2013).

\section{Figures}
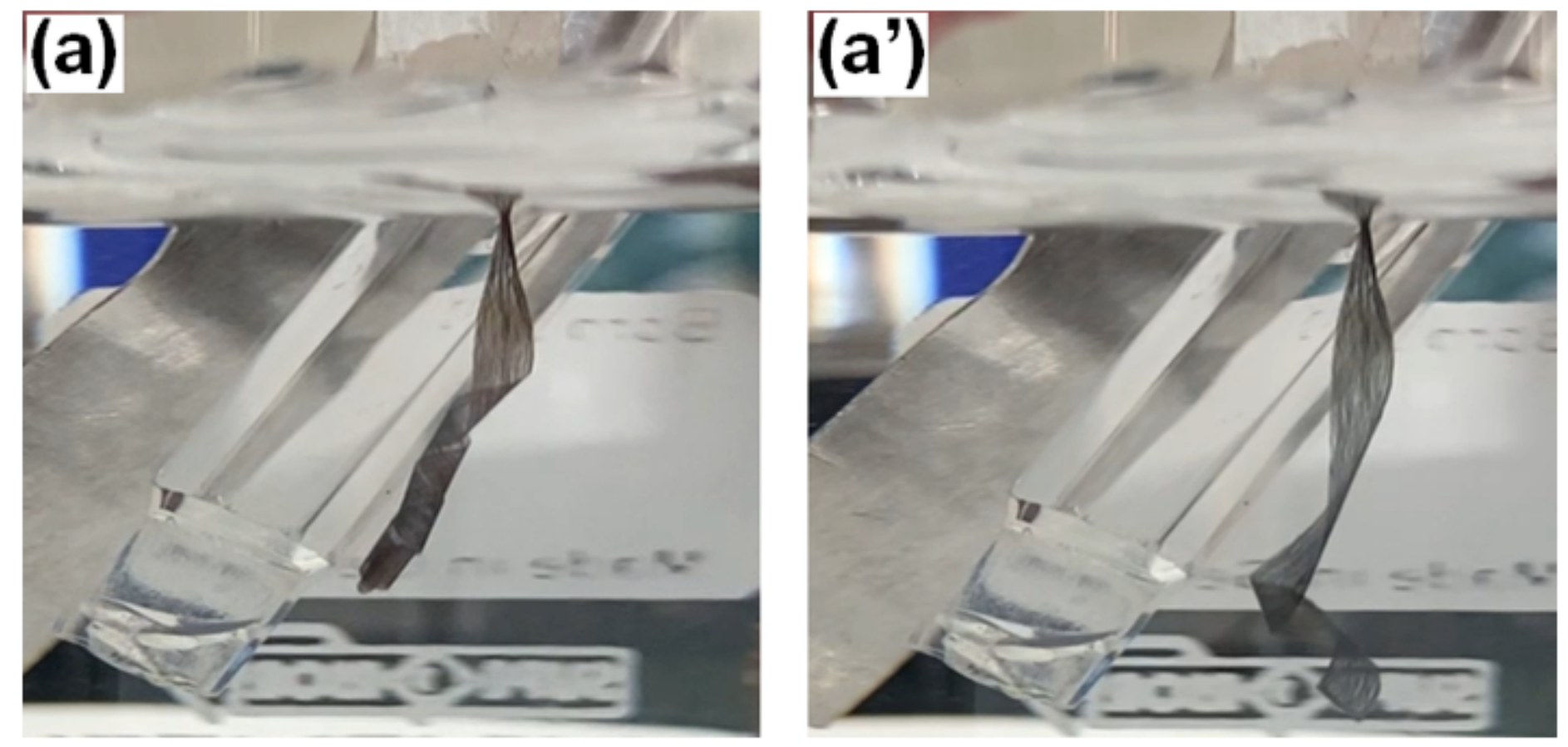

Figure 1 
Snapshots taken while applying a square potential to the material without attachment of any mass. The artificial muscle performs a reversible (a) clock-wise movement during reduction and a (a') anticlock-wise movement during the oxidation.
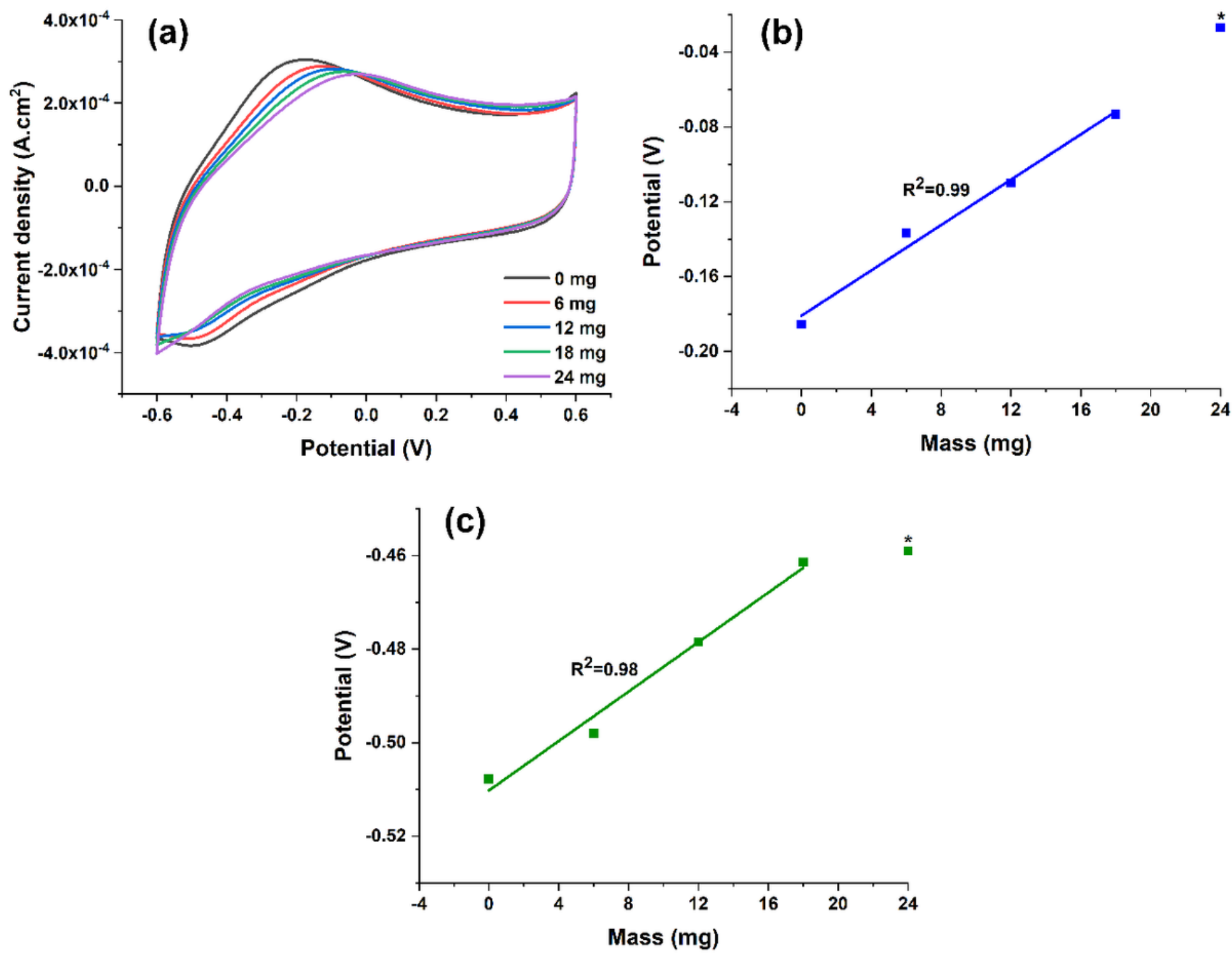

Figure 2

(a) Cyclic voltammograms recorded in $0.1 \mathrm{M} \mathrm{LiClO4}$ when various masses on the microribbon strip were clung; Variation of (b) anodic and (c) cathodic potentials as function of the attached mass. 

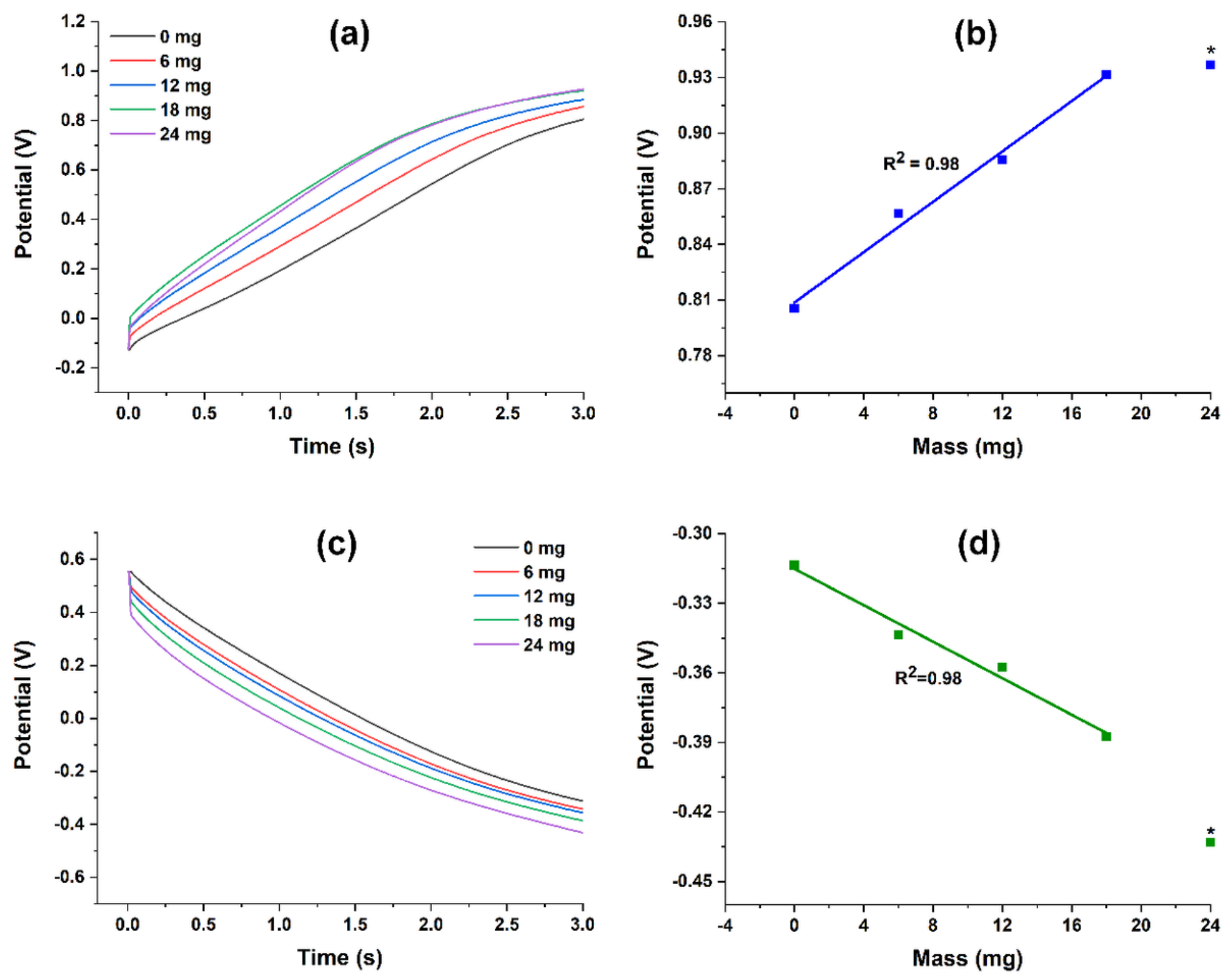

Figure 3

Evolution of the potential reached by the artificial muscle during application of a square current pulse of $\pm 0.5 \mathrm{~mA}$, obtaining the $(\mathrm{a}, \mathrm{b})$ anodic and $(\mathrm{c}, \mathrm{d})$ cathodic chronopotentiograms for each studied weight. 

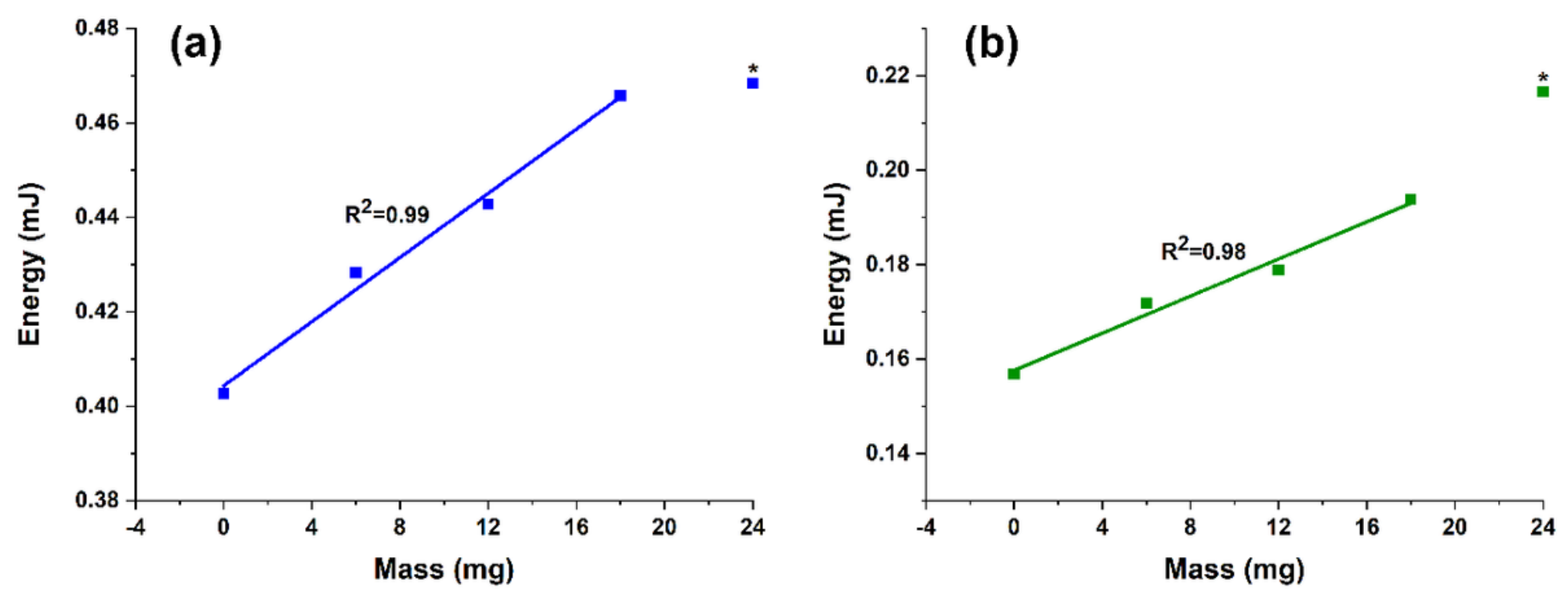

Figure 4

Energy consumed by the electrochemical reactions as function of the attached weight for (a) oxidation and (b) reduction processes.
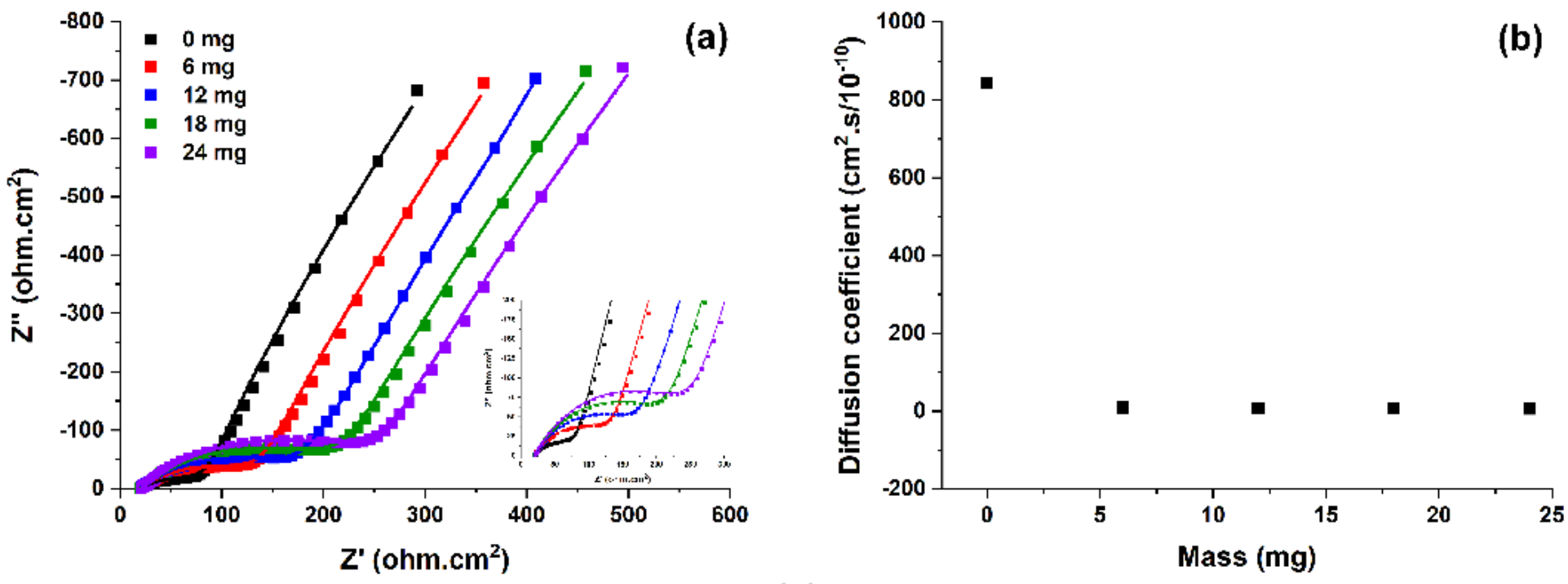

(c)

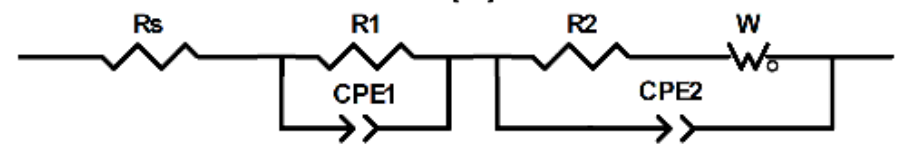

Figure 5

(a) Nyquist plots recorded by changing the clung weigh and (b) the corresponding diffusion coefficients estimated for each mass; (c) equivalent electrical circuit used for fitting the EIS results and determining the electrode parameters; Inset: zoom on the impedance semicircles. 


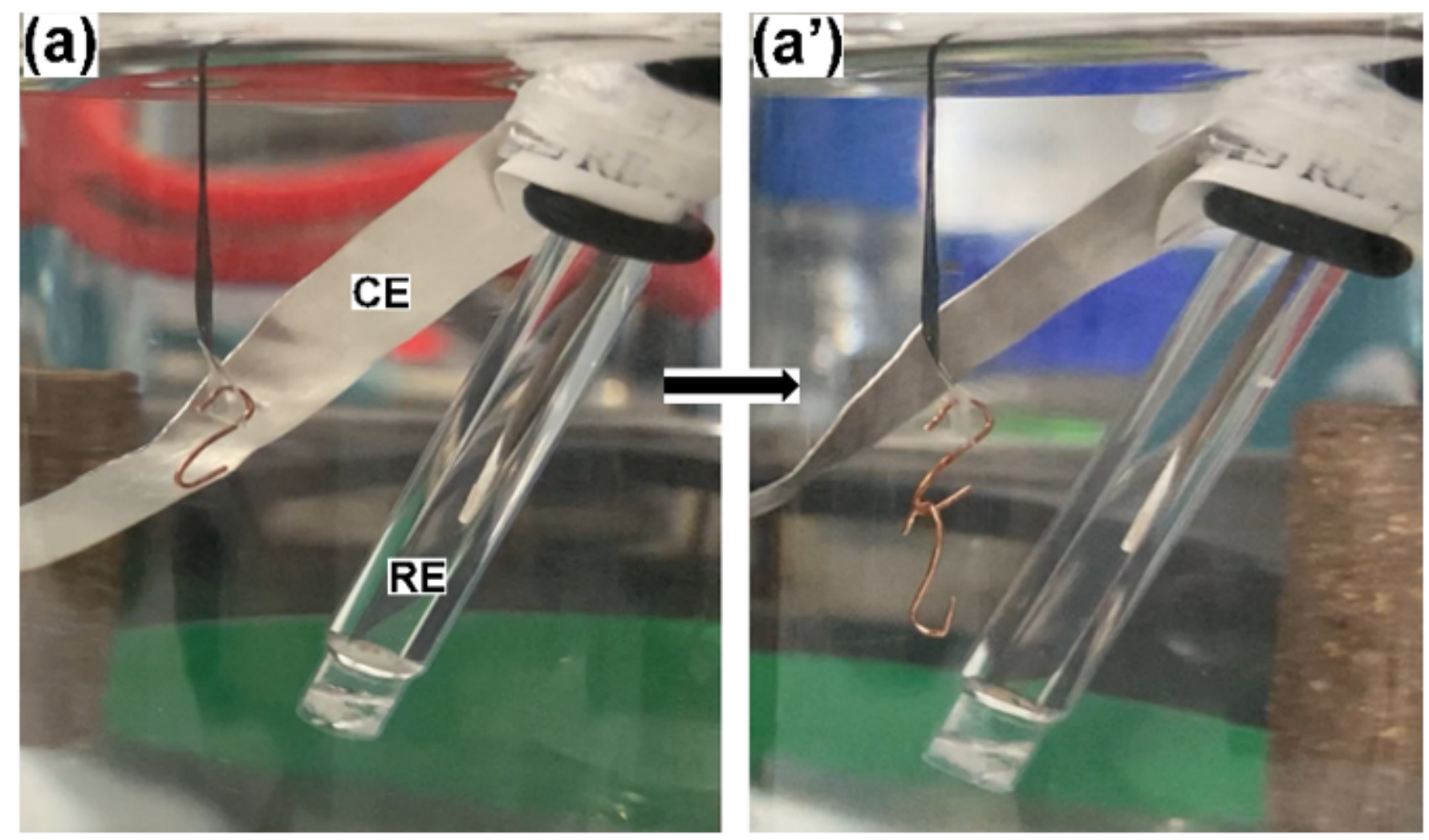

Figure 6

Experimental set-up used during characterization. An electrochemical cell containing an $\mathrm{Ag} / \mathrm{AgCl}$ reference electrode (RE), a platinum plate counter electrode (CE) and the PPy coated microribbon strip as working electrode holding (a) $6 \mathrm{mg}$ and (b) $12 \mathrm{mg}$.

\section{Supplementary Files}

This is a list of supplementary files associated with this preprint. Click to download.

- s1.mp4

- s2.mp4 